Supporting information

\title{
Multiscale Modeling of Sub-Entanglement-Scale Chain Stretching and Strain Hardening in Deformed Polymeric Glasses
}

\author{
Weizhong Zou ${ }^{\#}$, Soroush Moghadam ${ }^{+}$, Robert S. Hoy ${ }^{*^{*}}$, Ronald G. Larson ${ }^{\# * *}$ \\ \# Department of Chemical Engineering, University of Michigan, Ann Arbor, MI 48109 \\ + Department of Mechanical Engineering, University of Michigan, Ann Arbor, MI 48109 \\ $\ddagger$ Department of Physics, University of South Florida, Tampa, FL 33620 \\ *Correspondence: email: rlarson@umich.edu, phone: (734) 936-0772, FAX: (734) 763-0459 \\ **Correspondence: email: rshoy@usf.edu, phone: (813) 974-0063, FAX: (813) 974-5318
}

Effect of coarse-graining (CG) on parameters in HBD model. Here we discuss how the HBD parameters shown in Table I in the main text are affected by the level of coarse graining, i.e., the value of $N_{K, s p}$, which is the number of Kuhn steps represented by a single spring in the HBD simulations. From the Rouse model, the longest relaxation time of the polymer is,

$$
\tau^{p}=\zeta_{b} \frac{\left(N_{s p}+1\right) N_{K} b_{K}^{2}}{6 \pi^{2} k_{B} T}=\zeta_{b} \frac{\left(N_{s p}+1\right) N_{s p} N_{K, s p} b_{K}^{2}}{6 \pi^{2} k_{B} T} \equiv \alpha \tau^{s}
$$

where $\zeta_{b}$ is the drag coefficient per bead, $N_{s p}+1$ is the number of beads per chain in the HBD simulations ( $N_{s p}$ is the number of springs), and $b_{K}$ is the Kuhn length. The total number of Kuhn steps in the chain is $N_{K}=N_{s p} N_{K, s p}$ where $N_{K, s p}$ is the number of Kuhn steps per spring. $\tau^{p}$ and $\tau^{s}$ are the polymeric and the segmental relaxation times, respectively, and $\alpha$ is defined as the ratio $\tau^{p} / \tau^{s}$. Equation (S1) can be re-arranged to:

$$
\alpha=\frac{\zeta_{b}}{\tau^{s}} \frac{\left(N_{s p}+1\right) N_{s p} N_{K, s p} b_{K}^{2}}{6 \pi^{2} k_{B} T}
$$

Since the segmental relaxation time $\tau^{s}$ should be roughly independent of polymer chain length at a given temperature, and represents local relaxation at a length scale of order of a monomer, and since $\zeta_{b}$ is proportional to $N_{K, s p}$, the relaxation time of a Kuhn step is $\tau^{K} \sim \zeta_{b} b_{K}^{2} / N_{K, s p} k_{B} T$, we can estimate the value of $\alpha$ to be:

$$
\alpha \propto \frac{\tau^{K}}{\tau^{s}}\left(N_{s p}+1\right) N_{s p} N_{K, s p}^{2} \cong \alpha^{K} N_{K}^{2}
$$

where $\alpha^{K} \equiv \tau^{K} / \tau^{s}$. Thus, for a fixed polymer length, the value of $\alpha$ (taken in our simulations to be $8.0 \times 10^{4}$ ), remains constant regardless of the CG level. Note that for the chain simulated in the paper, $N_{K}=360$, and since the chain is flexible $\alpha^{K} \approx 1$, so that $N_{K}^{2}=1.3 \times 10^{5}$, close to the value of $\alpha$ used here. The values of other HBD parameters, namely, the total number of Kuhn steps $N_{K}$, the initial age of the glass or the so-called "waiting time" $t_{\mathrm{w}}$, the glassy modulus $G^{\mathrm{s}}$, and the deformation rate $\dot{\epsilon}$, reflect the chain length, the thermomechanical history of the glass, or other experimental conditions. Since both HBD and MD simulations start from the same initial chain 
conformations, the values of these parameters are unaffected by the coarse-graining level. This leaves two parameters, the polymeric modulus $G^{p}$ and the fluidity parameter $\mu$ to be set. In the formula for the polymeric stress used in Eq. (4a) of our previous paper, ${ }^{3} G^{p}$ is a "spring modulus":

$$
G^{p} \equiv \frac{3 \rho R T}{M_{S}} \sim N_{K, s p}^{-1}
$$

where $\rho$ is the mass density of the glass, and $M_{\mathrm{S}}$ is the molecular weight corresponding to a single spring, whose value is inversely proportional to the number of Kuhn steps per spring and hence to the CG level. (Note that in Ref. 3, Eq. (4b) gives the formula $G^{\mathrm{p}}=v k_{\mathrm{B}} T$, where $v$ had been incorrectly defined as the number of molecules per unit volume. However, $v$ must instead be taken to be the number of springs per unit volume, because the expression for stress in Eq. (4a) in Ref. 3 contains a sum over all springs in the denominator. Note also that the denominator in this expression in Eq. (4a) in Ref. 3 must contain a dot product of end-to-end vectors rather than an outer product so that the denominator is a scalar.) Thus, the value of $G^{\mathrm{p}}$ used here depends on the level of coarse graining since it is proportional to the stress contributed by a single spring.
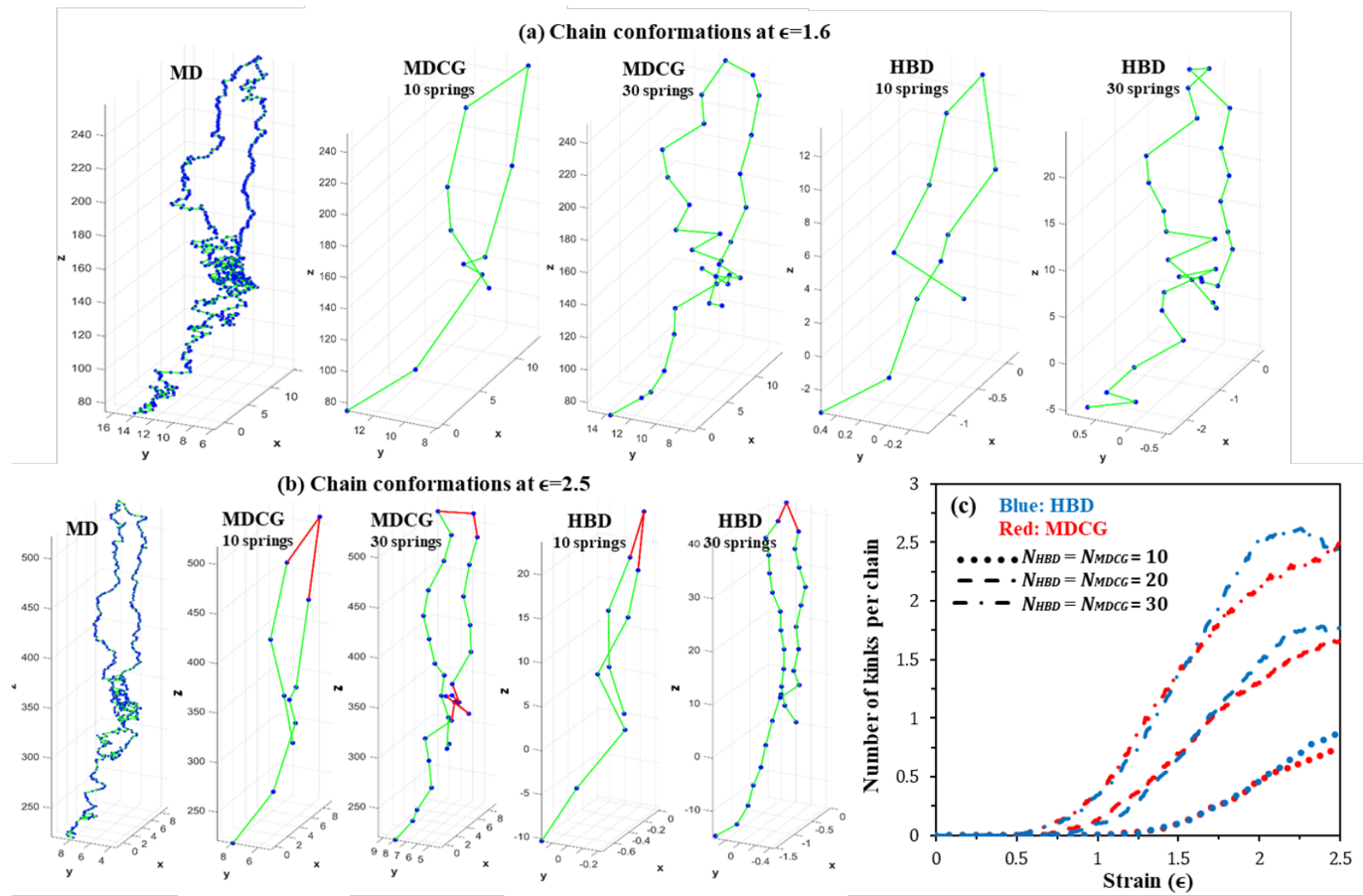

Figure S1. The same as Fig. 1 in the main text except that different levels of coarse-graining are used for HBD simulations with $N_{\mathrm{HBD}}=10$ and 30 springs at the corresponding values of $\mu=325,145$. and 75 , respectively. Note that the example chain taken here from the ensemble of MD chains is different chain than that taken in the main text, to further illustrate the agreement between the strain-dependent conformations of HBD and MD chains.

Hence, for chain conformations under asymptotically high deformation rate, with the identical initial glass state, i.e., the same values of $t_{\mathrm{w}}, G^{\mathrm{s}}, N_{\mathrm{K}}$, and $\alpha$ given above, the only parameter that 
can be readjusted to match with the results of MD simulation at different CG levels is $\mu$. The resulting excellent agreement in the chain conformations as well as the predicted number of kinks between HBD and MD simulations obtained by adjusting $\mu$ for different levels of CG is shown in Fig. S1.

Insensitivity of segmental stretch and fraction of highly stretched segments to chain resolution and rejuvenation parameter. We plot in Fig. S2 the average segmental stretch and fraction of highly stretched segments as functions of the applied strain for MD and HBD chains for three different values of $N_{\text {HBD }}$ and three different values of the rejuvenation parameter $\mu$. These plots show that both quantities are rather insensitive to $N_{H B D}$ and $\mu$, as was shown for the nonaffinity of deformation in Fig. 3 of the main text.
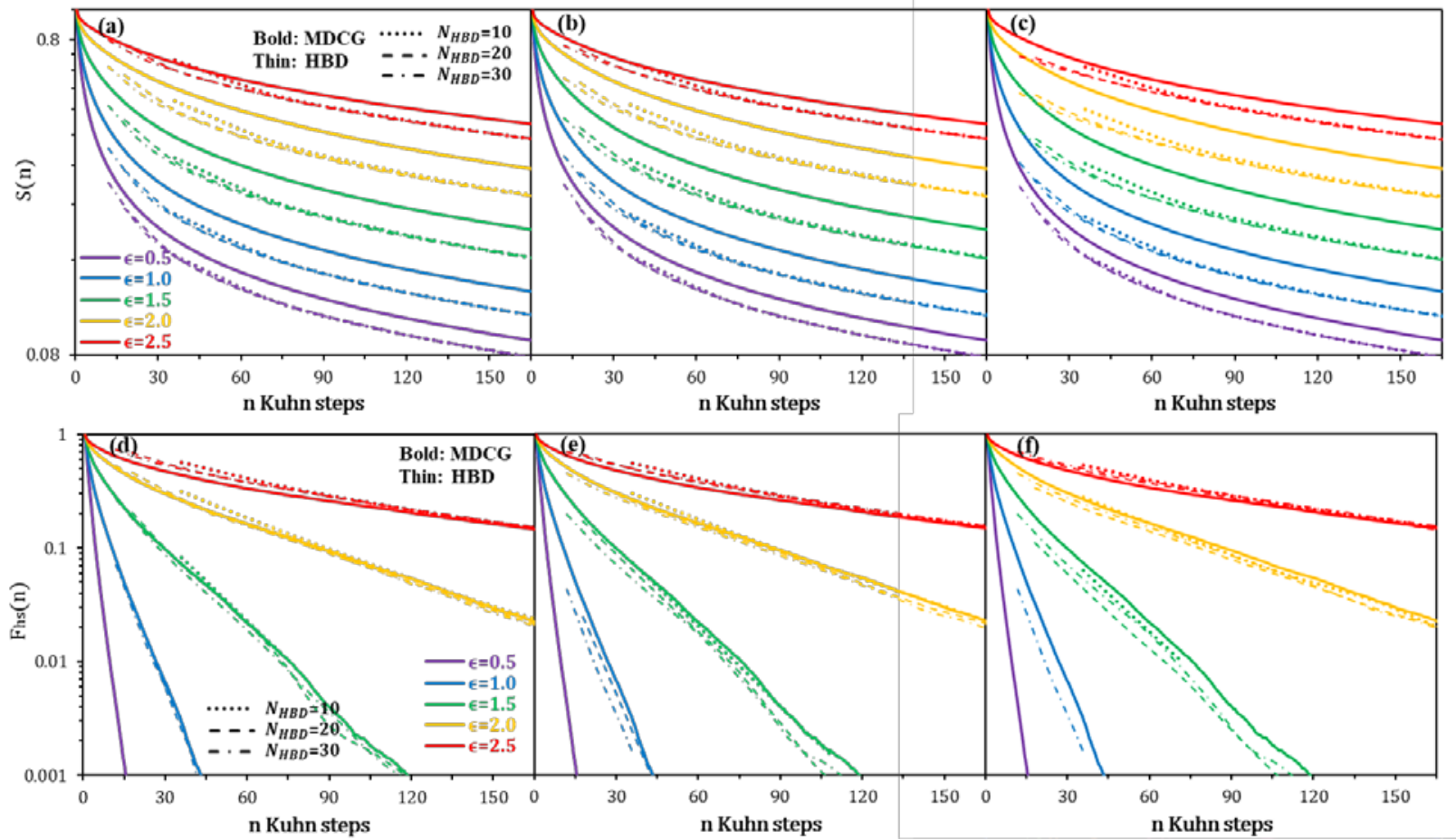

Figure S2. The mean segmental stretch $S(n)$ (a)-(c) as well as the fraction of "highly stretched segments" $F_{h s}(n)(\mathrm{d})$ (f) as functions of the imposed strain at various level of coarse-graining $N_{\text {HBD }}$ and rejuvenation parameter: for (a) and (d) $\mu=72$; for (b) and (e) $\mu=143$; and for (c) and (f) $\mu=286$. Response of fine-grained Kremer-Grest polymer model is shown with solid lines while that of the coarse-grained HBD model is depicted by dotted lines.

Agreement between HBD and MD for higher extension rate and uniaxial compression. By using the same value of parameters in Table I of main text, we here compare in Figs. S3-S6 the predicted chain properties between MD and HBD simulations under uniaxial extension at an orderof-magnitude higher as well as lower strain rate than that in the main text, and in Figs. S7-S8 the same properties under uniaxial compression at the same absolute rate used for uniaxial extension in the main text. The good agreements between the results of the two models for chain conformations and deformation affinity, chain extensibility, and the fraction of highly stretched segments, confirm the generality of our conclusions regarding the molecular origin of strain hardening and the ability of the same input parameters to provide similar agreement regardless of the applied strain rate and deformation type. 


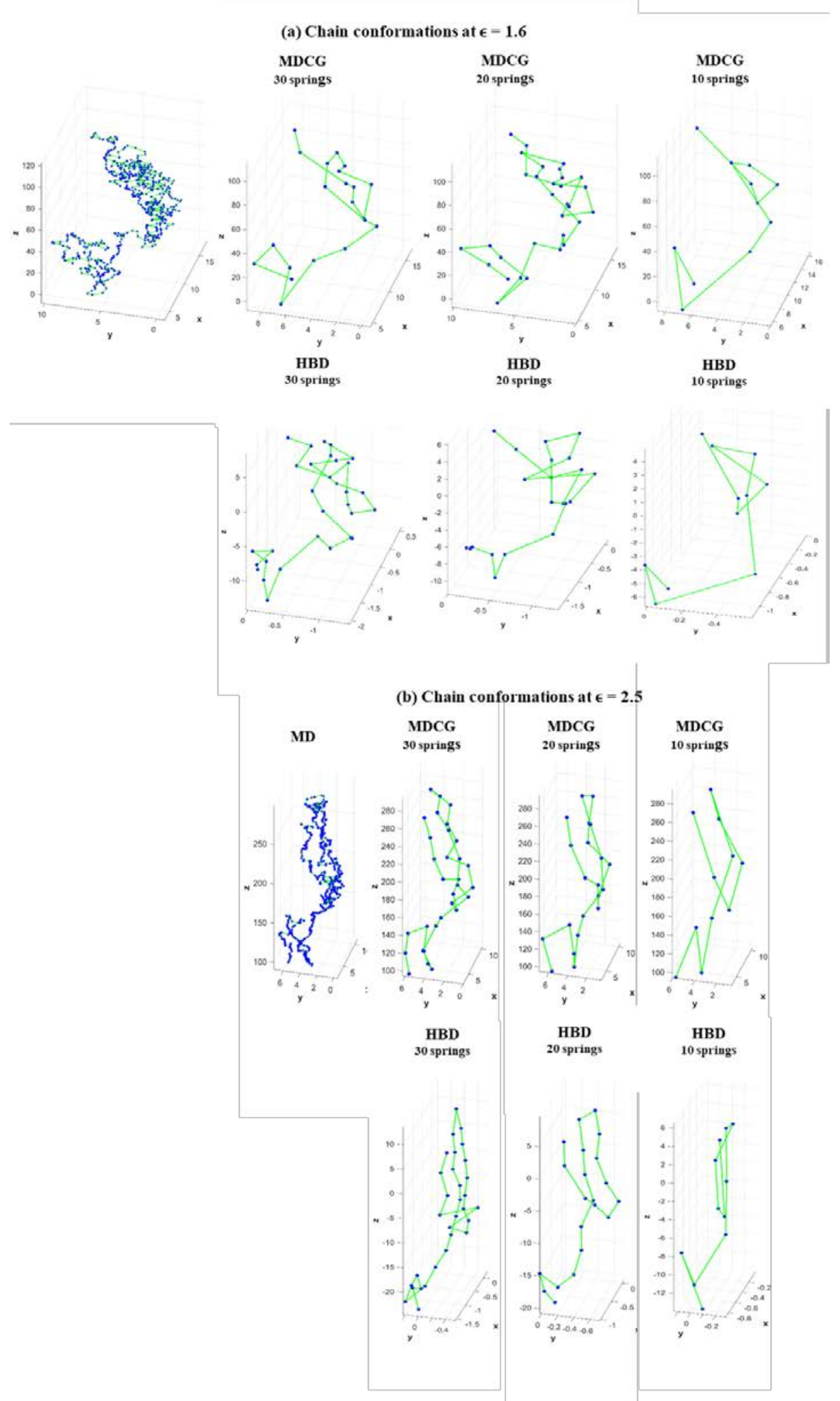

Figure S3. The same as Fig. S1 except that a 10 times higher strain rate is used for both HBD (at $\dot{\epsilon}=10^{-4} \mathrm{~s}^{-1}$ ) and MD ( $\dot{\epsilon}=2.5 \times 10^{-4}$ in LJ units) simulations. Note that in this and Figs. S4-S8 all the adjustable parameters are kept at the same values as those in Table I of main text. 

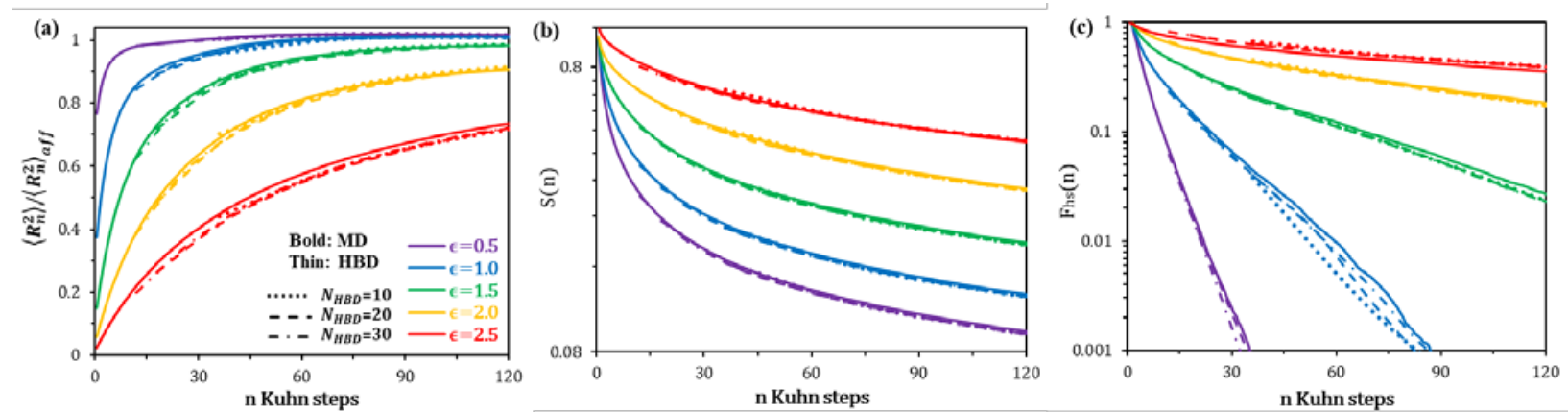

Figure S4. The same as Fig. 2 in the main text except that a 10 times higher strain rate is used for both HBD (at $\dot{\epsilon}=$ $\left.10^{-4} \mathrm{~s}^{-1}\right)$ and $\mathrm{MD}\left(\dot{\epsilon}=2.5 \times 10^{-4}\right.$ in LJ units) simulations. Note that results correspond to all three different levels of coarse-graining with $N_{\mathrm{HBD}}=10,20$, and 30 springs are also shown for HBD simulations at the corresponding values of $\mu=325,143$, and 75 , respectively. 

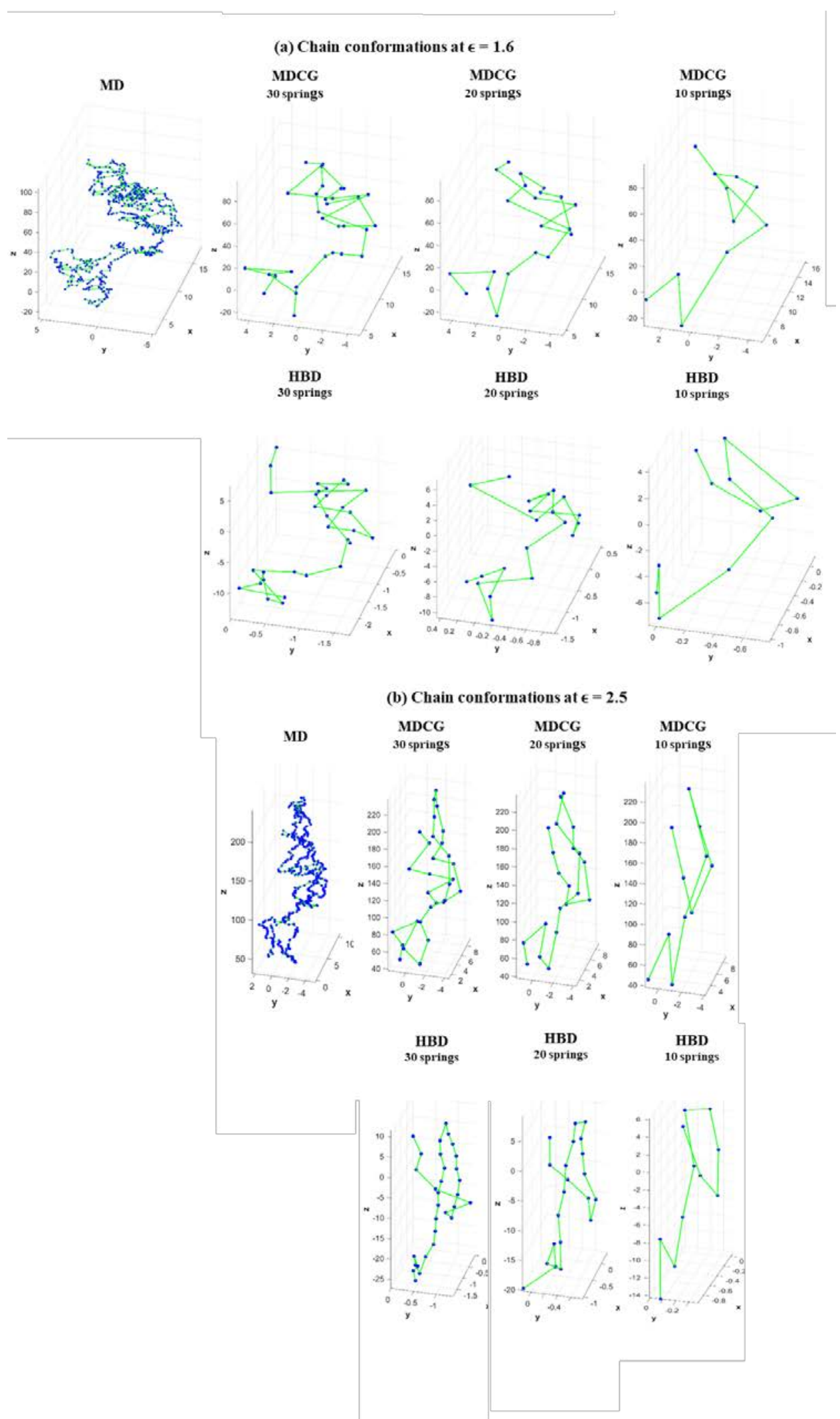

Figure S5. The same as Fig. S1 except that a 10 times lower strain rate is used for both HBD (at $\dot{\epsilon}=10^{-6} \mathrm{~s}^{-1}$ ) and MD ( $\dot{\epsilon}=2.5 \times 10^{-6}$ in LJ units) simulations. 

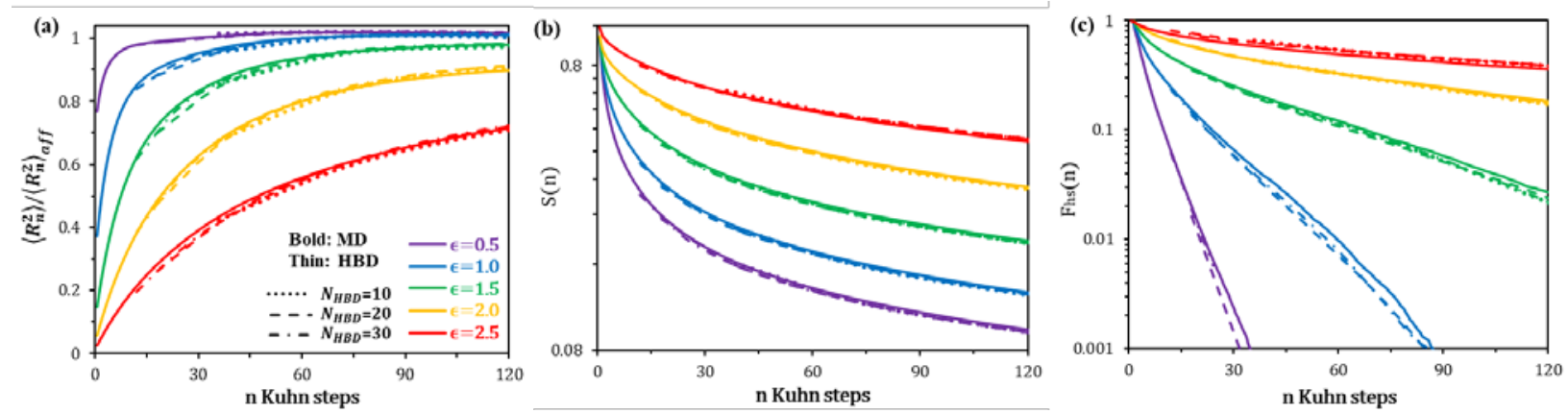

Figure S6. The same as Fig. S4 except that a 10 times lower strain rate is used for both HBD (at $\dot{\epsilon}=10^{-6} \mathrm{~s}^{-1}$ ) and MD $\left(\dot{\epsilon}=2.5 \times 10^{-6}\right.$ in LJ units) simulations.

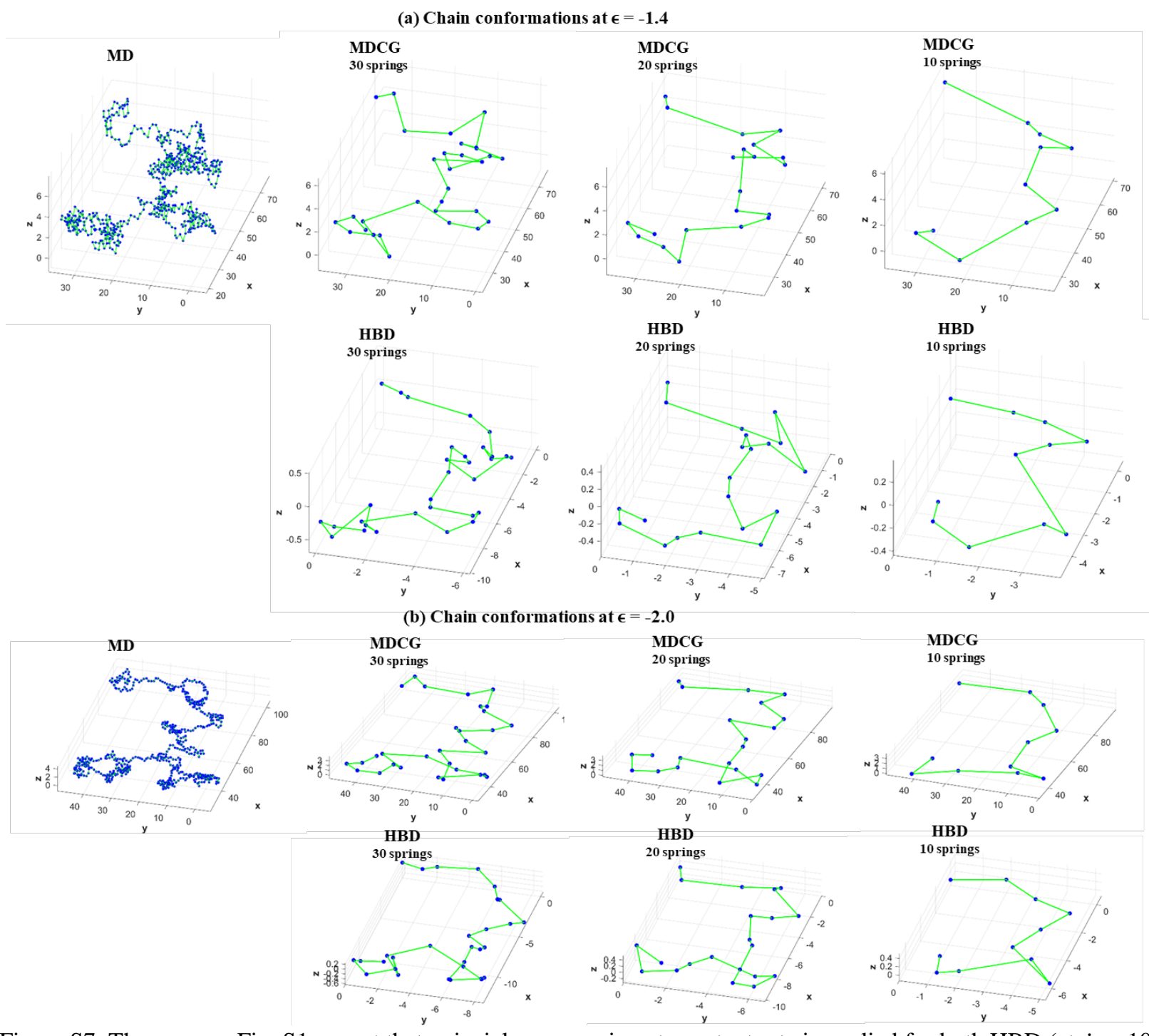

Figure S7. The same as Fig. S1 except that uniaxial compression at constant rate is applied for both HBD (at $\dot{\epsilon}=-10^{-}$ $\left.{ }^{5} \mathrm{~s}^{-1}\right)$ and $\mathrm{MD}\left(\dot{\epsilon}=-2.5 \times 10^{-5}\right.$ in LJ units) simulations. Extensional flow is made compressional by simply reversing the sign of the extension rate $\dot{\epsilon}$ from positive to negative. 

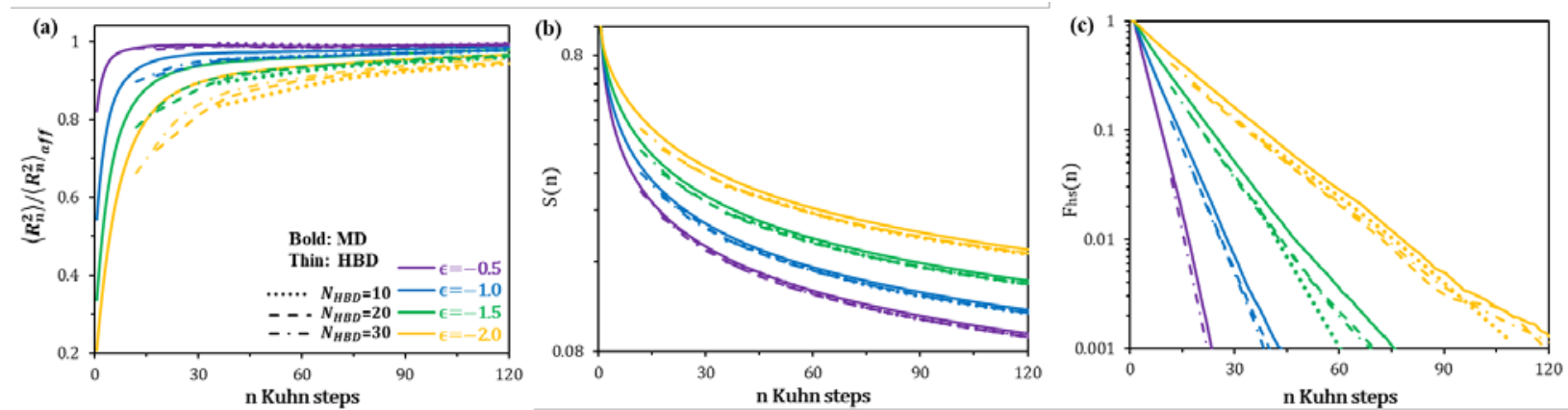

Figure S8. The same as Fig. S4 except that uniaxial compression at constant rate is applied for both HBD (at $\dot{\epsilon}=-10^{-}$ $\left.{ }^{5} \mathrm{~s}^{-1}\right)$ and $\mathrm{MD}\left(\dot{\epsilon}=-2.5 \times 10^{-5}\right.$ in LJ units) simulations.

Identification of kinks and entangled kink pairs. Since the HBD model is coarse-grained, to quantify the local formation of kinks and compare its predictions consistently with that from the fine-grained MD simulations, as discussed in the main text, MD chains need to be first coarsegrained into MDCG chains with the same number of MDCG vectors as there are springs in the HBD chains. Then the following procedure is taken to find "kinks" in both coarse-grained and fine-grained chain models. As illustrated by Fig. S9(a), the local formation of kinks in MD simulations is here identified by the condition that the two connecting vectors on each side of a kink point containing $N_{\text {conn }}$ beads must each have an angle relative to the deformation direction (i.e., z axis) of less than $\theta_{c}$, and a fractional stretch greater than $\lambda_{c}$. Note that $N_{c o n n}$ is the range of beads used to identify a kink-forming vector, (and thus in principle can be applied to every bead in the MD chain). This differs from the MDCG strands, which sequentially connect a set of $N_{M D} / N_{M D C G}$ springs from one end of the MD chain to the other. Thus, for fine-grained models, there are many more beads that can be considered as possible kink points than is the case for coarsegrained chain models (i.e., MDCG and HBD models), because the latter have fewer positions available as possible kink positions. The method used is as follows.
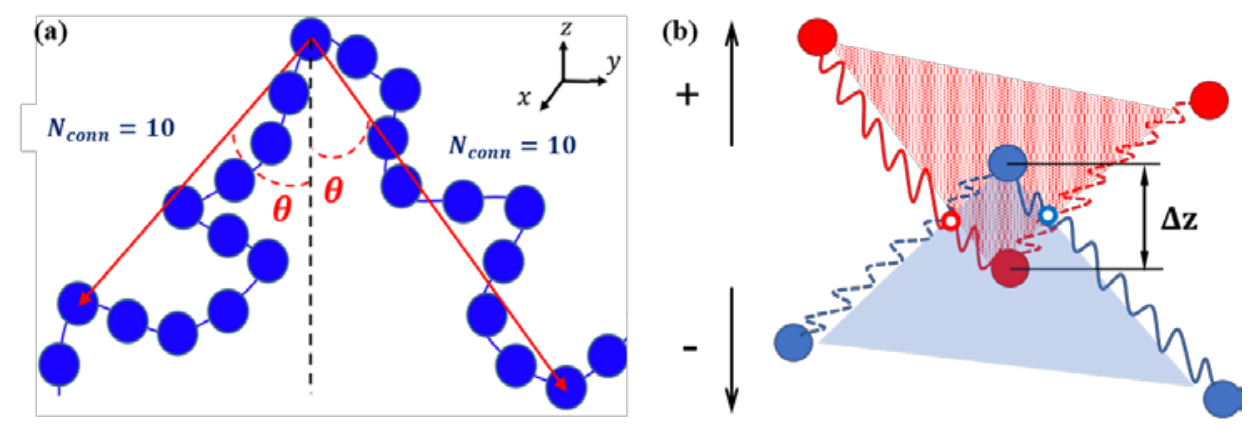

Figure S9. (a) Definition of "kink-forming" vectors used to identify local kinks in MD chain conformations without coarse graining. For illustration, we here use $N_{c o n n}=10$ as an example. (b) Schematic of how an entangled kink is determined in MD simulations using the kink's connecting vectors defined in panel (a).

1. First, we examine all beads in a given chain and check whether they can be considered as "potential” kink points. To qualify as a "potential” kink point, the coordinates of the bead along the deformation axis (i.e., the $z$ axis) must satisfy $\left(z_{i}-z_{i-1}\right)\left(z_{i}-z_{i+1}\right)>0$, where $i$ is the bead index of the fine-grain MD model which varies from 1 to $N$.

2. After choosing a potential kink-point bead, say bead $i$, we find the positions of beads $i+N_{\text {conn }}$ and $i-N_{\text {conn, }}$, respectively, where $N_{\text {conn }}$ is the number of beads included in a strand bounding 
each side of the potential kink point. Joining each of the positions of bead $i+N_{\text {conn }}$ and of $i$ $N_{\text {conn }}$ to that of the potential kink bead $i$, we create the two connecting vectors of the kink, as illustrated in Fig. S9(a).

3. We compute the relative stretch of each of these connecting vectors and the angle that each makes with the deformation axis.

4. To confirm bead $i$ as a kink position, we require that the fractional stretch of both connecting vectors exceed a value $\lambda_{c}$ and that both connecting vectors make an angle smaller than $\theta_{c}$ with the deformation axis.

All "potential" kink points that satisfy the above criteria are accepted as actual kink points. To apply the above procedure to coarse-grained chains, since each connecting vector of the coarsegrained chain already represents multiple Kuhn steps, we apply the above procedure, but with $N_{\text {conn }}=1$. After finding beads at which $\left(z_{j}-z_{j-1}\right)\left(z_{j}-z_{j+1}\right)>0$ is satisfied, with $j$ the index of the beads in the coarse-grained chain, one needs to check directly if the connecting vectors satisfy the $\lambda_{c}$ and $\theta_{c}$ conditions. A seemingly obvious way to identify "entangled kink pairs" is to perform primitive path analyses ${ }^{11,12}$ and find pairs of contacting primitive paths that satisfy some predefined kink criterion. However, some recent work has indicated that it is more accurate to regard chains in deformed polymer glasses as being constrained by their rheological tubes rather than by individual, crosslink-like entanglements. ${ }^{13}$ More importantly, we wish to find entanglements that are localized at kinks. Thus, the following criteria are used to identify the entanglements that are localized at kinks:

1. The two kinks have opposite polarity, as illustrated by Fig. S9(b).

2. The two kinks are "intertwined" with each other. This is determined by defining a "kink triangular surface" as the surface bounded by the two kink-forming connecting vectors of each kink and by a third vector formed by linking the free ends of the two connecting vectors. If the two kink triangular surfaces formed by two kinks intersect each other, and only one of the endto-end vectors of a kink intersects the triangular surface of the other, then the two kinks are considered entangled.
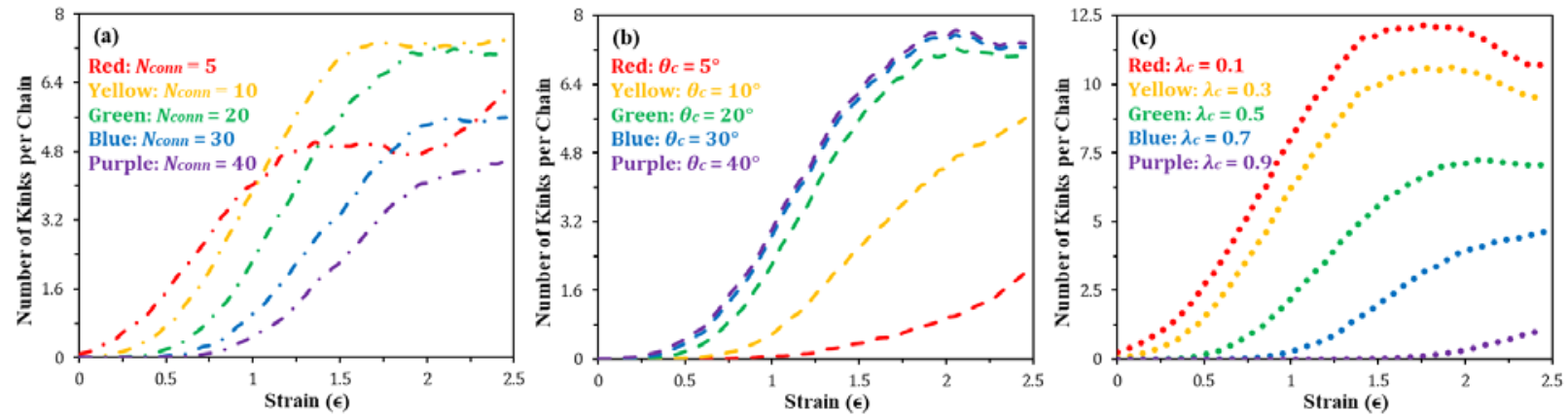

Figure S10. Sensitivity of the number of kinks per chain in the fine-grained MD simulations on (a) $N_{\text {conn, }}$ (b) $\theta_{\mathrm{c}}$, and (c) $\lambda_{c}$. The default values of parameters are $N_{\text {conn }}=20, \theta_{c}=20^{\circ}$, and $\lambda_{c}=0.5$, unless stated otherwise.

Using $N_{\text {conn }}=20, \lambda_{c}=0.5$ and $\theta_{c}=20^{\circ}$ gives the results in Figs. 3 and 4 in the main text. Here we also report the sensitivity of the number of kinks per chain in MD simulations to the kink identification parameters, namely $\theta_{c}, N_{\text {conn }}$ and $\lambda_{c}$. Figure S10(a) shows that by increasing the value of $N_{\text {conn }}$ to find the connecting vectors of the kinks in the MD simulations, the number of kinks per 
chain initially rises and then drops again, with identical plateau values for $N_{\text {conn }}=10$ and 20 . This is expected since by increasing $N_{\text {conn }}$ beyond a threshold, the connecting vectors of the potential kink point eventually extend beyond the region of a single kink and therefore no longer satisfy the angle criterion. We therefore chose $N_{\text {conn }}=20$ for our MD analysis in the main text. Figure S10(b) shows that increasing $\theta_{c}$ initially results in an increase in the number of kinks in the MD simulations; however, increasing it beyond $\sim 20^{\circ}$ no longer affects the number of kinks, unless the angle exceeds $40^{\circ}$, in which case it is no longer reasonable to consider the conformation to be that of a "kink," which is intended to be a relatively sharp fold of the chain back on itself along the flow direction. Based on these results, $\theta_{c}=20^{\circ}$ was chosen as the critical limiting angle limit for defining a kink in the figures main text. Finally, we study the effect of stretch ratio of the kink's connecting vectors on $N_{\text {conn. }}$. As expected, as the minimum stretch ratio, $\lambda_{c}$, gets larger, the number of kinks decreases. Since in this manuscript, we are considering locally formed kinks at strains below that needed for the chain to reach the fully "kinked" or folded state, ${ }^{1,10}$ we choose a minimum stretch ratio of 0.5 to define a locally stretched state for the two strands bounding the kink.

Insensitivity of numbers of kinks to strain rate. The number of kinks for different strain rates for the MD model and the HBD model at different strain rates is shown in Fig. S11. Note that for uniaxial extension, strain rate has almost no effect on either the number of kinks or of entangled kinks, but that in uniaxial compression, the number of kinks is much reduced.
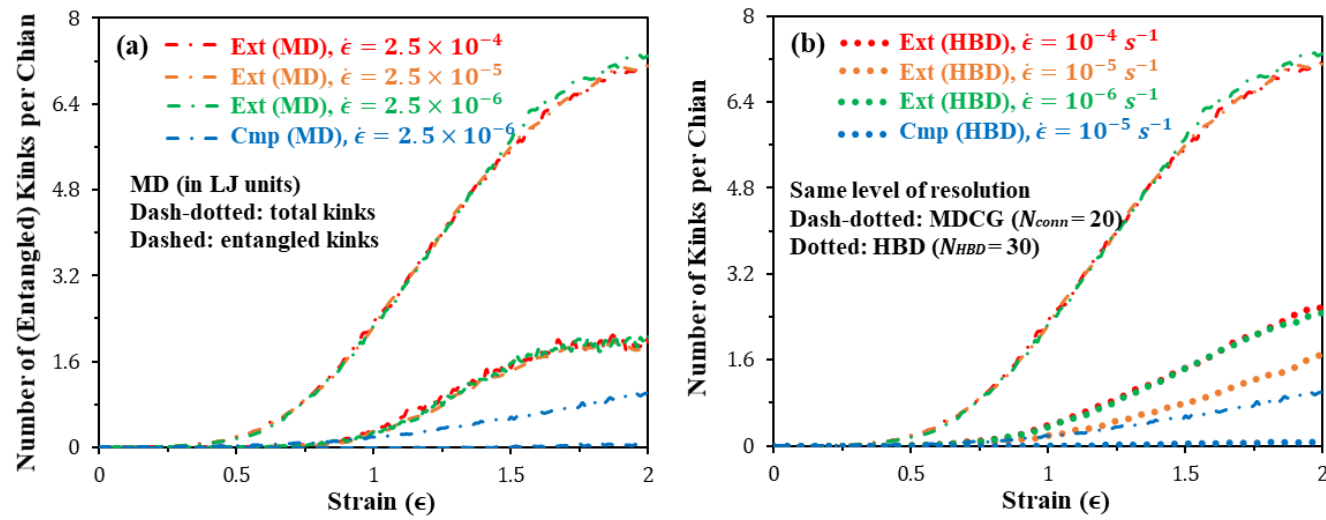

Fig. S11 (a) The total number of kinks per chain vs entangled ones as a function of imposed strain in the fine-grained MD simulations using default values of parameters for kink identification, i.e., $N_{\text {conn }}=20, \theta_{c}=20^{\circ}$, and $\lambda_{c}=0.5$. (b) Average numbers of kinks per chain as a function of imposed strain for MDCG and HBD models, where the value of $N_{\text {conn }}=20$ is chosen so that $N_{M D} / N_{\text {conn }}$ matches the values of resolution $N_{M D C G}=N_{H B D}=30$ used in the coarse-grained HBD and MDCG models. The labels "Ext" and "Cmp" correspond to extensional and compression flows, respectively and we neglect the negative signs for extensional strain rates and strains used in earlier figures.

Drawbacks of single-mode segmental model. Although it allows semi-quantitative fits to the experimental data of Lee et al., ${ }^{4,5}$ the single-mode Maxwell model for segmental dynamics with characteristic time controlled by a simple fluidity equation has major weaknesses. Figure S12 shows, in particular, that phenomenological fluidity equation (see Eq. (1) in the main text) in this model is too crude to predict accurately the change in segmental mobility under a constant deformation rate. In fact, as shown by the inserted plot in Fig. S12(a), the fluidity model greatly underestimates the drop in $\tau_{p r b}^{s}$ at strains less than 0.025 during the period of rising stress in the start-up deformation, while overestimating the decrease in $\tau_{p r b}^{S}$ at a strain of around 0.025 , near 
the maximum in stress. As a result, the decay in stress after the maximum is reached is much faster than in the experiments. ${ }^{6,7}$ (Notice that for the purpose of comparison, the linear elastic deformation, i.e., strain below $1 \%$, as well as the height of stress overshoot predicted by different versions of segmental models were fitted to the experiments through the choice of the glassy modulus $G^{\text {s }}$ and waiting time $t_{\mathrm{w}}$.) This deficiency in modeling the evolution of segmental relaxation time can, however, be fixed easily by the use of the experimentally measured $\tau_{p r b}^{S}$ from fluorescence spectroscopy. After reoptimizing the values of some parameters, the corresponding new simulation prediction, shown in Fig. S12(b), gives a stress-strain curve that is in much better agreement with experiment. However, this new prediction still shows somewhat stronger strainsoftening than the experimental data, due to the single time-constant segmental relaxation assumed here. Further improvement requires using a stretched exponential relaxation of segmental mobility as is observed experimentally. ${ }^{4,5}$ Using a stretched exponential, represented by three modes, leads to the results shown in Fig. 7 of the main text.

In addition to the above issues regarding segmental dynamics, like most constitutive models, the values of model parameters are not set a priori, but are fitted to the measured data. Thus, an inadequate physical model for segmental dynamics can also result in uncertainties and errors in the values of parameters obtained by fitting experimental data, especially when the data is rich in features. Since the applied deformation in Fig. S12(b) extends only into the strain-softening regime, the stress contributed from the polymeric mode remains mostly inactivated, and only the initial age of the sample $t_{w}\left(=40000 \mathrm{~s}\right.$ or $11.1 \mathrm{~h}$, see Table S1) and the glassy shear modulus $G^{s}$ (= $0.5 \mathrm{GPa}$, see Table S1) are relevant and were obtained by matching the simulation predictions with the experimental data. Relative to the multi-mode empirical model discussed in main text (see Fig. 7), $G^{s}$ is roughly halved in the single mode fluidity model, while $t_{w}$ is increased by a factor of four. Thus, in the single-mode fluidity model, the best-fit values of parameters deviate from ranges that reflect the physical properties of a young glass with $G^{s}$ around $1 \mathrm{GPa}$ and $t_{w}$ a few hours long. This further supports the importance of stretched exponential relaxation for development of a more rigorous physics-based segmental model.
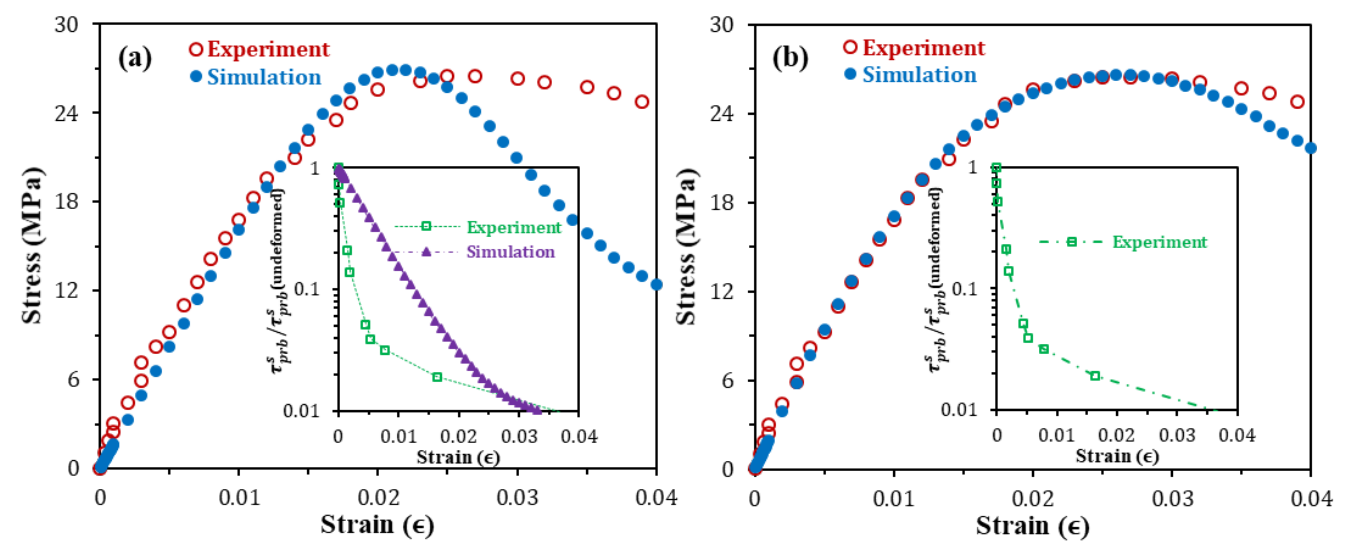

Figure S12. The same experimental data as in Fig. 7(b) of the main text fitted by the HBD model with single-mode segmental dynamics with relaxation time given by (a) simple fluidity equation (filled purple triangles in the inserted plot); (b) direct measurement from fluorescence spectroscopy (hollow green squares in the inserted plot). Note that in contrast to values used in the multi-mode HBD model in the main text (Fig. 7(b)) given in the third line of Table S1, the values of $t_{\mathrm{w}}$ and $G^{\mathrm{s}}$ used in Fig. S12 are given in the second line of Table S1. 
Table S1. Values of segmental parameters for single- and multi-mode HBD simulations in Fig. S12 and Fig. 7(b) with segmental relaxation time from experiment or from the simple fluidity model.

\begin{tabular}{c|cc|ccc}
\hline \hline Figures & No. of modes & Segmental time & $\boldsymbol{G}^{\mathbf{s}}(\mathbf{M P a})$ & $\boldsymbol{t}_{\mathbf{w}}(\mathbf{h})$ & $\boldsymbol{\mu}$ \\
\hline Fig. S12(a) & 1 & Fluidity equation & 550 & 5.8 & 143 \\
Fig. S12(b) & 1 & Experiment & 500 & 11.1 & N/A \\
Fig. 7(b) & 3 & Experiment & 800 & 5.8 & N/A \\
\hline \hline
\end{tabular}

Note that the 3-mode segmental dynamics were achieved by decomposing the KWW function through the modified genetic algorithm with the nonexponentiality $\beta$ determined from the experiment (see the section below).

Decomposition of KWW function through the Genetic Algorithm (GA). Here we present our procedure for decomposing the KWW function with known characteristic time $\tau_{p r b}^{s}$ and stretching exponent $\beta$ from fluorescence spectroscopy into a set of single exponential relaxation terms using optimization with a genetic algorithm (GA). This is necessary to obtain the constants used in the multi-mode segmental relaxation model given by Eq. (2) in the main text. Since a generalized protocol for fitting an arbitrary time-dependent function with a discrete spectrum of monoexponentials has been elaborated in Ref. 2, in what follows we focus on utilizing this protocol for our case without a detailed discussion of the GA method itself.

The GA is used to generate the times constants $\tau_{j}^{S}$ and prefactors $C_{\mathrm{j}}$ in Eq. (2) of the main text for a set of exponential functions whose sum represents adequately the stretched-exponential function $\exp \left[-\left(x / \tau_{p r b}^{S}\right)^{\beta}\right]$ with given values of $\tau_{p r b}^{S}$ and $\beta$. We first generate a set of values of the stretched exponential function at times spaced logarithmically over a finite time interval whose upper boundary $\left(t_{\max }\right)$ is determined by the following criterion,

$$
\exp \left[-\left(\frac{t_{\text {max }}}{\tau_{\text {prb }}^{S}}\right)^{\beta}\right]=1 \%, t_{\min } / t_{w}=\frac{t_{w}}{t_{\max }}
$$

Note that the lower boundary $\left(t_{\mathrm{min}}\right)$ is chosen so that these boundaries are symmetric on the logarithmic scale. Thus, the ratio $t_{\max } / t_{\min }$ represents the width of the spectrum of the decomposed single exponential terms, which depends on the value of $\beta$ since the spectrum narrows as $\beta$ increases. By normalizing the characteristic times for each exponential mode by $\tau_{\text {prb }}^{S}$, which is equivalent to setting $\tau_{p r b}^{s}=1$, we were able to use the GA to create a library of output values of $\tau_{j}^{S} / \tau_{p r b}^{S}$ and $C_{\mathrm{j}}$ for values of $\beta$ varying from 0.2 to 0.75 . With this library, the amplitude $C_{\mathrm{j}}$ and the normalized time constant $\tau_{j}^{S} / \tau_{\text {prb }}^{S}$ for each mode $j$ can be retrieved at any desired time by fitting to the stretched exponential function in Eq. (2b) in the main text, upon inputting $\beta$. Thus, the parameters $C_{\mathrm{j}}$ and $\tau_{j}^{S} / \tau_{\text {prb }}^{S}$ change with time as required by the time-dependent values of $\beta$ and $\tau_{\text {prb }}^{s}$ from the experimental data. We carried out the fitting for three different numbers of exponential modes, i.e., $M=3$, 4, and 5 and results are shown in Fig. S13 with their corresponding fitting errors (defined in Eq. (S6)) given in Fig. S14. 

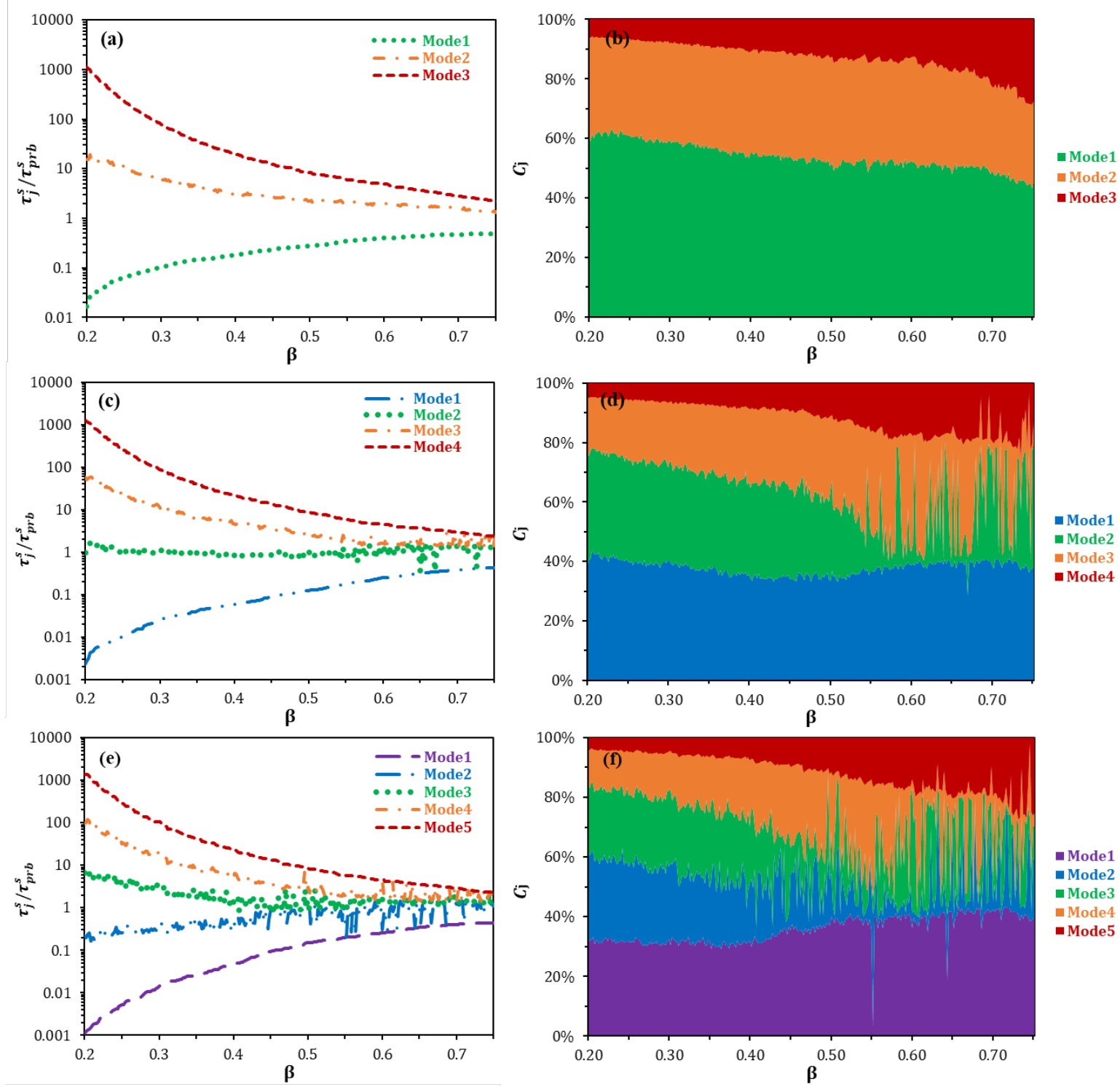

Figure S13. The normalized characteristic times $\tau_{j}^{s} / \tau_{p r b}^{s}$ in (a), (c), and (e) with corresponding amplitudes $C_{\mathrm{j}}$ in (b), (d), and (f) obtained from the GA as functions of $\beta$ at three different total numbers of exponential modes: (a) and (b) for $M=3$; (c) and (d) for $M=4$; (e) and (f) for $M=5$.

$$
\varepsilon_{k}=\frac{1}{N} \sum_{i=1}^{N}\left\{\frac{\left|\exp \left[-\left(\frac{x_{i}}{\tau_{p r b}^{S}}\right)^{\beta_{k}}\right]-\sum_{j=1}^{M} C_{j} \exp \left(-\frac{x_{i}}{\tau_{j}^{S}}\right)\right|}{\exp \left[-\left(\frac{x_{i}}{\tau_{p r b}^{S}}\right)^{\beta_{k}}\right]}\right\}
$$

Here, $\varepsilon_{\mathrm{k}}$ is the averaged relative error when using $M$ different mono-exponentials to represent the KWW function of $\beta \mathrm{k}$. The subscripts " $i$ " and " $j$ " stand, respectively, for the $i$ th data point at time $x_{\mathrm{i}}$ and the $j$ th mode with amplitude $C_{\mathrm{j}}$ and normalized time constant $\tau_{j}^{S} / \tau_{p r b}^{S}$. The subscript " $k$ " is the index for the set of values of $\beta_{\mathrm{k}}$, from smallest to largest, in the library. An empirical constraint (see Eq. (S7)) is also introduced so that the smallest and the largest values of $\tau_{j}^{S} / \tau_{p r b}^{S}$, respectively, 
increase and decrease with increasing $\beta$ values. This is done to smooth out the fluctuations of $\tau_{j}^{S} / \tau_{p r b}^{S}$ as $\beta$ increases.

$$
\left\{\begin{array}{l}
\max \left[\tau_{j}^{S}\left(\beta_{k}\right)\right] \geq \max \left[\tau_{j}^{S}\left(\beta_{k+1}\right)\right] ; \\
\min \left[\tau_{j}^{S}\left(\beta_{k}\right)\right] \leq \min \left[\tau_{j}^{S}\left(\beta_{k+1}\right)\right] ;
\end{array} \quad j=1, \cdots, M\right.
$$

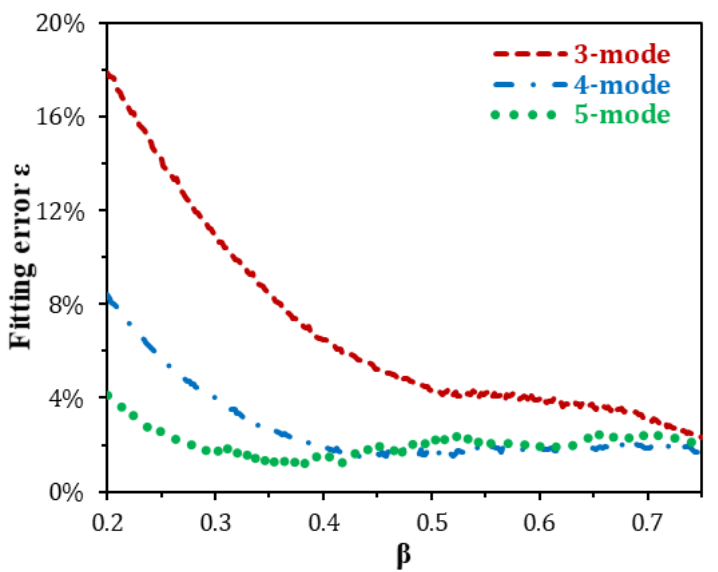

Figure S14. The relative error in representing the KWW function with different numbers of mono-exponential terms with amplitude and characteristic time optimized by a GA.

According to Figs. S13 and S14, using more exponential terms reduces the fitting error for small $\beta$ values with broad relaxation spectra on one hand, but loses the distinction in $\tau_{j}^{S} / \tau_{\text {prb }}^{S}$ between different modes for large $\beta$ values with narrow spectra on the other hand. Since the relaxation time spectrum narrows down effectively to a nearly single-Maxwell model at large deformation as $\beta$ increases to unity, the latter situation is more troublesome under the creep measurements, for which the fluctuations in $C_{\mathrm{j}}$ would induce a significant level of noise in deformation rate, therefore making quantitative prediction rather challenging. Thus, we choose a constant value $M=3$ as a trade-off between accuracy and robustness of the results. Note that since $\beta$ is time-dependent, a constant value of $M$ is needed to avoid adding and removing modes during a simulation.

Empirical correlation between segmental mobility and stretching exponent $\boldsymbol{\beta}$. The lack of quantitative data limits the development of a heterogeneity model, since it requires experimental data for the segmental relaxation time $\tau_{p r b}^{s}$ and its stretching exponent $\beta$, and these are frequently not experimentally inaccessible. Recent findings for polymeric glasses indicate that the heights of barriers between local energy minima in the potential energy landscape (PEL) become larger as the system moves towards the lower-energy regions of the PEL, suggesting that the heterogeneity, and therefore the stretching exponent $\beta$, can be linked to the segmental mobility. ${ }^{8,9}$ For example, the thermally excited sampling of the PEL should be especially retarded in lower-energy regions of the PEL due to their higher barriers, thus leading to a more pronounced heterogeneity and hence smaller $\beta$. Inspired by the plot of stretching exponent $\beta$ vs. the dimensionless segmental relaxation time $\tau_{\text {prb }}^{S} / t_{\mathrm{w}}$ in Lee et al. ${ }^{4,5}$ we show in Fig. S15 that a crude "master curve" can be obtained empirically, whose mathematical expression is given by Eq. (S8), by extracting $\beta$ and $\tau_{p r b}^{S} / t_{\mathrm{w}}$ from multiple measurements of fluorescence spectroscopy by Ediger and coworker, ${ }^{4-7}$ at various temperatures, type of probes, preparation history, as well as deformation processes. 


$$
\beta=0.25-0.61 \ln \left(\frac{\tau_{p r b}^{s}}{t_{w}}\right)
$$

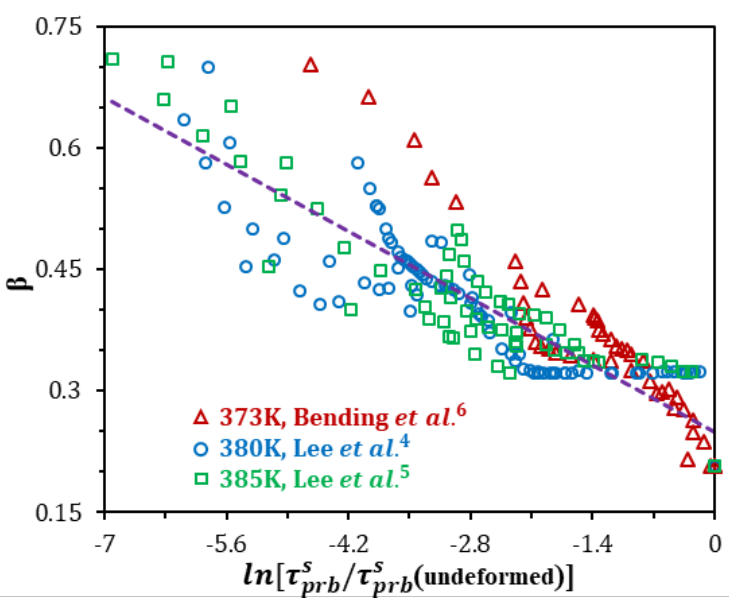

Figure S15. Correlation between the enhanced segmental mobility $\tau_{p r b}^{s} / t_{\mathrm{w}}$ and stretching exponent $\beta$ from results of fluorescence spectroscopy in Refs. ${ }^{4-6} R^{2}$ value for the dashed line fit, given by Eq. (S8) is 0.755 .

This correlation between segmental mobility and heterogeneity, in Fig. S15, offers us an empirical route to incorporate multi-mode segmental dynamics into the HBD model, as summarized below:

$$
\begin{aligned}
& \left\{\begin{array}{c}
\dot{\boldsymbol{\sigma}}_{j}^{S}+\mathbf{v} \cdot \boldsymbol{\nabla} \boldsymbol{\sigma}_{j}^{S}=\boldsymbol{\sigma}_{j}^{S} \cdot \boldsymbol{\nabla} \mathbf{v}+(\boldsymbol{\nabla v})^{T} \cdot \boldsymbol{\sigma}_{j}^{S}-\left(\boldsymbol{\sigma}_{j}^{S}-\boldsymbol{I}\right) / \tau_{j}^{S} \\
\boldsymbol{\Sigma}^{S}=G^{S} \sum_{j}^{M} C_{j}\left(\boldsymbol{\sigma}_{j}^{S}-\boldsymbol{I}\right) \\
\exp \left[-\left(\frac{x}{\tau_{p r b}^{S}}\right)^{\beta}\right]=\sum_{j}^{M} C_{k} \exp \left(-\frac{x}{\tau_{j}^{S}}\right)
\end{array}\right. \\
& \left\{\begin{array}{c}
\beta=0.25-0.61 \ln \left(\frac{\tau_{p r b}^{S}}{t_{w}}\right) \\
\dot{\tau}_{p r b}^{S}=1-\mu \sqrt{2 \operatorname{tr}(\boldsymbol{D}: \boldsymbol{D})}\left(\tau_{p r b}^{s}-\tau_{0}^{s}\right) \\
\left.\tau_{p r b}^{S}\right|_{t=0}=t_{w}
\end{array}\right.
\end{aligned}
$$

By comparing Fig. S16 with Fig. 1 of the main text, it is evident that the generalization of the simple fluidity model to the 3-mode empirical segmental model using Eq. (S9) does not degrade the excellent agreement between the chain conformations predicted by the HBD and MD simulations. 

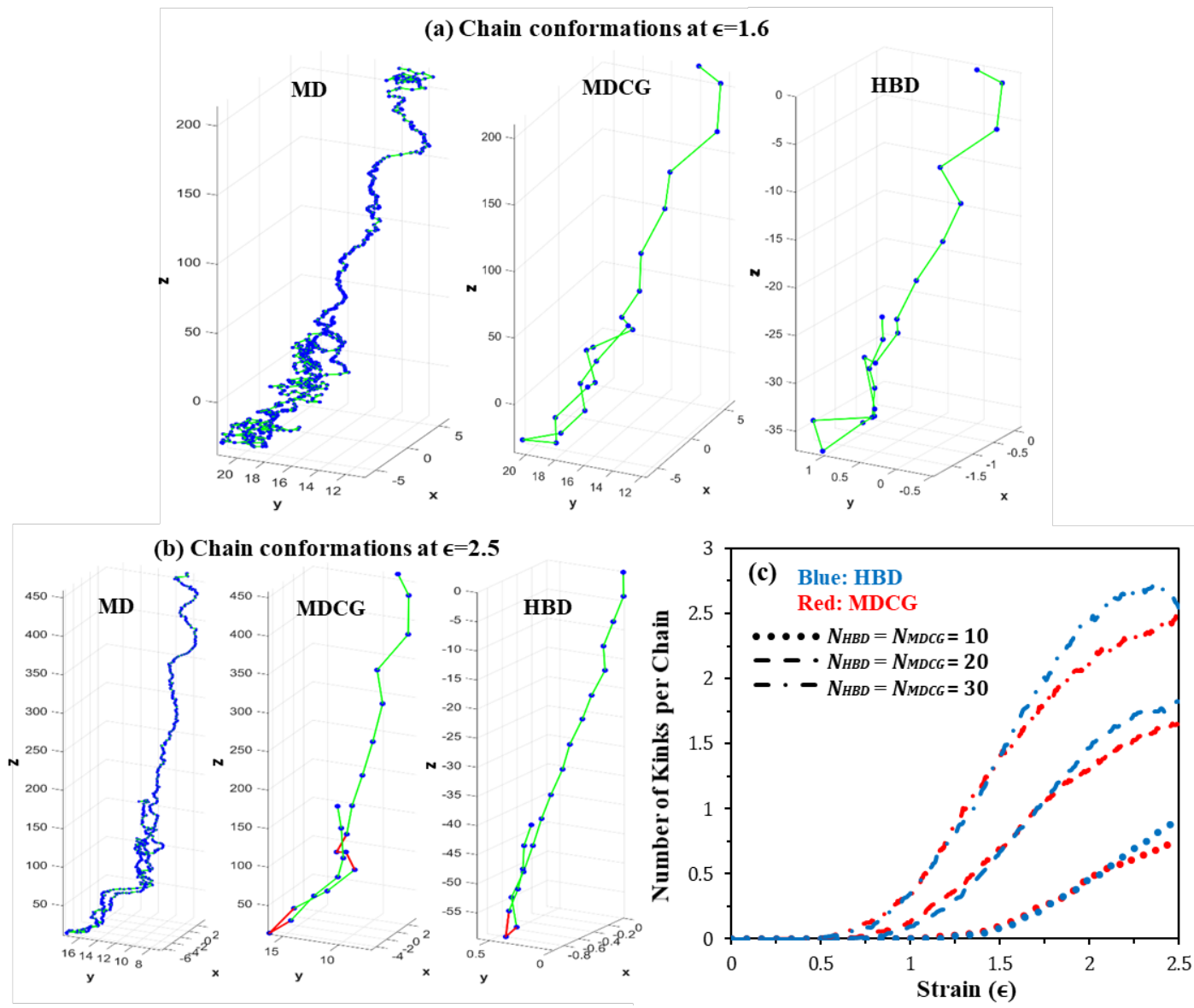

Figure S16. (a, b). The same as Fig. S1(a, b) except that the 3-mode empirical segmental model is used for HBD simulation using $N_{\mathrm{HBD}}=N_{\mathrm{MDCG}}=20$, and $\mu=143$. (c) The same as Fig. S1(c), with the corresponding values of $\mu$ given in the caption to Fig. S1. Other parameters are also the same as those of Fig. S1.

\section{References}

[1] Moghadam, S.; Dalal, I. S.; Larson, R. G. Slip-link and kink dynamics models for fast extensional flow of entangled polymeric liquids, Polymer, 2019, 11, 465.

[2] Zou, W.; and Larson, R. G. A mesoscopic simulation method for predicting the rheology of semi-dilute wormlike micellar solutions. J. Rheol. 2014, 58, 681-721.

[3] Zou, W.; and Larson, R. G. A hybrid Brownian Dynamics/constitutive model for yielding, aging, and rejuvenation in deforming polymeric glasses. Soft Matter 2016, 3, 3853-3865.

[4] Lee, H.-N.; Paeng, K.; Swallen, S. F.; et al. Molecular Mobility of Poly(methyl methacrylate) Glass During Uniaxial Tensile Creep Deformation. J. Polym. Sci. B 2009, 47, 1713-1727.

[5] Lee, H.-N.; Paeng, K.; Swallen, S. F.; and Ediger, M. D. Direct measurement of molecular mobility in actively deformed polymer glasses. Science 2009, 323, 231-234.

[6] Bending, B.; and Ediger, M. D. Comparison of mechanical and molecular measures of mobility during constant strain rate deformation of a PMMA glass. J. Polym. Sci. B 2016, 54, 1957-1967.

[7] Lee, H.-N.; Riggleman, R. A.; de Pablo, J. J.; and Ediger, M. D. Deformation-induced mobility in polymeric glasses during multistep creep experiments and simulations. Macromolecules 2009, 42, 4328-4336.

[8] Chung, Y. G.; and Lacks, D. J. Atomic mobility in a polymer glass after shear and thermal cycles. J. Phys. Chem. B 2012, 116, 14201-14205.

[9] Chung, Y. G.; and Lacks, D. J. How deformation enhances mobility in a polymer glass. Macromolecules 2012, 45, 4416-4421. 
[10] Moghadam, S.; Dalal, I. S.; Larson, R. G. Unraveling dynamics of entangled polymers in strong extensional flows. Macromolecules 2019, 52, 1296-1307.

[11] Hoy, R. S.; Foteinopoulou, K.; Kröger, M. Topological analysis of polymeric melts: Chain-length effects and fast-converging estimators for entanglement length. Phys. Rev. E 2009, 80, 031803.

[12] Tzoumanekas, C; Theodorou, D. N.; Topological analysis of linear polymer melts: a statistical approach. Macromolecules 2006, 39, 4592.

[13] Ge, T.; Tzoumanekas, C.; Anogiannakis, S. D.; et al. Entanglements in glassy polymer crazing: cross-links or tubes? Macromolecules 2017, 50, 459-471. 